\title{
Preoperative hypoalbuminemia was associated with acute kidney injury in high- risk patients following non-cardiac surgery: a retrospective cohort study
}

Nan Li ${ }^{1,2}$, Hong Qiao', Jing-Fei Guo ${ }^{3}$, Hong-Yun Yang ${ }^{4}$, Xue-Ying $\mathrm{Li}^{5}$, Shuang-Ling $\mathrm{Li}^{1,2^{*}}$ (D), Dong-Xin Wang ${ }^{1}$ and Li Yang ${ }^{2,6^{*}}$

\begin{abstract}
Background: Acute kidney injury (AKI) is a common complication following non-cardiac surgery with adverse short- and long- term morbidity and mortality. Evidence shows that hypoalbuminemia is associated with increased AKI risk in patients with infectious diseases and cancer and following cardiac surgery and transplant surgery. However, little evidence is available on non-cardiac surgery population. Thus, we investigated the association between preoperative hypoalbuminemia and AKI following non-cardiac surgery.

Methods: We retrospectively assessed perioperative risk factors and preoperative serum albumin concentration in 729 consecutive adult patients who underwent non-cardiac surgery from July 1, 2017, to June 30, 2018. Each patient was categorized according to maximal Kidney Disease Improving Global Outcomes criteria based on creatinine changes and urine output within the first week after surgery. Multivariate Logistic regression models were used to analyze the association between preoperative hypoalbuminemia and postoperative AKI.
\end{abstract}

Results: Of 729 patients, 188 (25.8\%) developed AKI. AKI incidence was higher in patients with preoperative serum albumin $<37.5 \mathrm{~g} / \mathrm{L}$ than in those with preoperative serum albumin $\geq 37.5 \mathrm{~g} / \mathrm{L}[35.9 \%$ (98/273) vs. 19.7\% (90/456), $P<0.001]$. Multivariate logistic regression analysis showed that preoperative serum albumin $<37.5 \mathrm{~g} / \mathrm{L}$ (odds ratio 1.892; 95\% confidence interval 1.238-2.891; $P=0.003$ ) was independently associated with postoperative AKI. Patients with preoperative serum albumin $<37.5 \mathrm{~g} / \mathrm{L}$ tended to have a higher but not significant ratio in AKI stage 2 ( $2.6 \%$ vs $1.1 \%, P=0.144)$ and much higher ratio in AKI stage $3(4.8 \%$ vs $0.7 \%, P<0.001)$ than those with preoperative serum albumin $\geq 37.5 \mathrm{~g} / \mathrm{L}$. AKI patients had a higher in-hospital mortality rate $[6.9 \%(13 / 188) \mathrm{vs} .0 .2 \%$ $(1 / 541), P<0.001]$. Kaplan-Meier analysis revealed that the cumulative survival rate decreased with increasing AKI severity $(P<0.001)$. Postoperative AKI was also associated with other worse outcomes, such as prolonged mechanical ventilation $[53.4(33.0,73.8)$ vs $14.7(11.1,18.3)$ hours, $P<0.001]$, intensive care unit stay $[4.0(3.1,4.9)$ vs $2.0(1.8,2.3)$ days, $P<0.001]$, postoperative hospital stay $[17.8(14.8,20.9)$ vs $12.3(11.3,13.3)$ days, $P<0.001]$, and higher total cost $[13,453(8538,20,228)$ vs $11,306(6277,16,400)$ dollars, $P<0.001]$.

Conclusions: Preoperative hypoalbuminemia was independently associated with AKI after non-cardiac surgery, and postoperative AKI was associated with poor outcomes.

Keywords: Hypoalbuminemia, Acute kidney injury, Non-cardiac surgery, Prognosis

\footnotetext{
* Correspondence: lishuangling888@hotmail.com; li.yang@bjmu.edu.cn

${ }^{1}$ Department of Critical Care Medicine, Peking University First Hospital, No.8

Xishiku Street, Xicheng District, Beijing 100034, China

${ }^{2}$ Critical Care Nephrology Research Center, Peking University First Hospital,

No.8 Xishiku Street, Xicheng District, Beijing 100034, China

Full list of author information is available at the end of the article
}

(c) The Author(s). 2019 Open Access This article is distributed under the terms of the Creative Commons Attribution 4.0 International License (http://creativecommons.org/licenses/by/4.0/), which permits unrestricted use, distribution, and reproduction in any medium, provided you give appropriate credit to the original author(s) and the source, provide a link to the Creative Commons license, and indicate if changes were made. The Creative Commons Public Domain Dedication waiver (http://creativecommons.org/publicdomain/zero/1.0/) applies to the data made available in this article, unless otherwise stated. 


\section{Background}

Acute kidney injury (AKI) is a common complication after non-cardiac surgery with an incidence ranging from 6.8 to $39.3 \%$ according to different patient populations [1, 2]. Several underlying susceptibilities, procedures, or exposures have been identified to be risk factors of postoperative AKI occurrence, such as older age, chronic kidney disease, diabetes, sepsis, major surgery, and hemodynamic instability [3]. Recent evidence demonstrated that AKI was independently associated with longer length of hospital stay and higher rate of 30day hospital readmission, 1-year end-stage renal disease, and mortality with more severe stage of AKI relating to poorer outcomes after non-cardiac surgery [4, 5]. Unfortunately, an effective treatment for AKI in the intensive care unit has not been established [6], strongly suggesting that early recognition of and adjusting for risk factors would be beneficial for high-risk patients.

Hypoalbuminemia is a well-established risk factor for increased morbidity and mortality in acutely ill patients [7]. The association between hypoalbuminemia and AKI is consistently evident in many observational studies conducted across different clinical settings, mainly focusing on infectious diseases, cancer, cardiac surgery, and transplant surgery [8-12]. Although the underlying mechanisms for this association are not fully elucidated, serum albumin may play a protection role in the maintenance of renal perfusion, preservation of proximal tubular integrity and function, binding of endogenous toxins and nephrotoxic drugs, prevention of oxidative damage, and delivery of protective lysophosphatidic acid [13, 14]. Therefore, we assumed that preoperative hypoalbuminemia might be associated with an increased risk of AKI following non-cardiac surgery. However, limited data are currently available on this topic [15-18]. Thus, this study aimed to investigate the association between preoperative serum albumin concentration and AKI occurrence in high-risk patients following non-cardiac surgery.

\section{Methods}

\section{Ethics and consent}

Ethical approval (2018-137) was provided by the Clinical Research Ethics Committee of Peking University First Hospital on July 4, 2018. Because of the retrospective nature of the study and no patient follow-up was performed, the ethics committee agreed to waive written informed consent. This study was performed in accordance with Strengthening the Reporting of Observational Studies in Epidemiology (STROBE) criteria [see Additional file 1: Table S1].

\section{Patients}

The study period was from July 1, 2017, to June 30, 2018, with the following inclusion criteria: (1) adult patients (age $\geq 18$ years), (2) undergoing non-cardiac surgery, (3) admitted to the surgical intensive care unit (SICU), and (4) at a high risk of postoperative AKI. High-risk patients referred to as patients having at least one of the following conditions: (1) preoperative comorbidities, including hypertension, diabetes mellitus, coronary heart disease, congestive heart failure, cerebrovascular disease, chronic kidney disease, lung disease, or liver disease; (2) major surgery, defined as surgery duration $\geq 2 \mathrm{~h}$; (3) ongoing organ dysfunction, defined as the sequential organ failure assessment (SOFA) score $\geq 2$ from one single organ system. Patients with any of the following criteria were excluded: (1) chronic kidney disease stage 5 or requiring long-term dialysis; (2) surgery involving kidney, such as nephrectomy, partial nephrectomy, nephroureterectomy, or kidney transplantation; (3) AKI events before surgery; and (4) incomplete clinical data. Our medical center is a teaching hospital affiliated with a university, which provides tertiary care and has about 1600 beds.

\section{Definitions of outcomes}

The primary end point was postoperative AKI development. Postoperative AKI and its severity was defined according to Kidney Disease Improving Global Outcomes criteria using the maximal change in serum creatinine compared with the preoperative baseline values and urine output during the first 7 postoperative days [19].

The secondary end points were the postoperative use of mechanical ventilation (MV) and its duration, length of ICU and postoperative hospital stay, number of postoperative complications other than AKI, total cost, and in-hospital mortality.

Other main postoperative complications were pulmonary infection, pleural effusion, pulmonary atelectasis, respiratory failure, surgical bleeding, new-onset arrhythmia, acute myocardial infarction, congestive heart failure, hemodynamic insufficiency, stroke, acute liver injury, disseminated intravascular coagulation, ileus, anastomotic leakage, intra-abdominal abscess, wound infection, wound dehiscence, urinary tract infection, sepsis, digestive tract bleeding, and venous thromboembolism [see Additional file 2: Table S2].

\section{Other data collection}

Patients' data were searched through the electronic medical records system of our hospital. Perioperative data were collected including demographic characteristics (age, sex), body mass index (BMI), medical history, American Society of Anesthesiology (ASA) physical status classification, as well as preoperative nephrotoxin exposure. Other data collected were preoperative clinical laboratory data, such as hemoglobin, albumin, baseline serum creatinine, and B-type natriuretic peptide (BNP). 
Intraoperative data included type and duration of surgery, emergency surgery, duration of anesthesia, maximal lactate, minimal hemoglobin, estimated blood loss, use of vasopressors, volume of artificial colloids infusion, and fluid balance. Postoperative data before AKI included new onset of nephrotoxin exposure, sepsis, use of vasopressors, minimal hemoglobin, maximal lactate and $\mathrm{BNP}$, perioperative blood transfusion, and non-renal SOFA score within $24 \mathrm{~h}$ of ICU admission.

\section{Statistical analysis}

Preoperative serum albumin concentration were firstly compared between patients with and without AKI by independent samples t-test. Then to determine its cutoff value for postoperative AKI occurrence, receiver operating characteristic curve analysis was performed. The patients were divided into two groups according to the occurrence of AKI or hypoalbuminemia. Quantitative variables with normal distribution were compared by independent samples t-test; numeric data with abnormal distribution were compared by Mann-Whitney $U$ test. Qualitative variables were compared by chi-squared test or Fisher's exact test. Time-to-event data were analyzed with Kaplan-Meier survival analysis, with difference between groups compared by log- rank test. After testing for collinearity, perioperative variables with $P<0.10$ and number of events $>10$ in the univariate analyses for AKI occurrence were included in multivariate logistic regression model (backward stepwise method) to identify independent risk factors for AKI. Furthermore, baseline variables unbalanced between patients with or without hypoalbuminemia were entered into propensity score matching. Then, patients were matched 1:1 based on their scores using nearest-neighbor matching with the tolerance being 0.02 . Thereafter, logistic regression analysis was performed to find out the association between hypoalbuminemia and AKI. Two-sided $P$ values $<0.05$ were regarded as statistically significant. The SPSS v21.0 software package was used for statistical processing. (SPSS Inc., Chicago, IL, USA).

\section{Results}

During the study period, a total of 971 patients at a high risk of AKI undergoing non-cardiac surgery were admitted to SICU; among them, 729 met the inclusion/exclusion criteria and were included in the final statistical analysis (Fig. 1). Baseline and perioperative data were listed in Tables 1 and 2. Among the enrolled patients, 188 (25.8\%) developed postoperative AKI stages 1, 2, and 3, which accounted for 21.9, 1.6, and 2.2\%, respectively. Patients with postoperative AKI had significantly lower level of preoperative albumin compared with patients without AKI $(36.7 \pm 6.3$ vs $39.3 \pm 6.0 \mathrm{~g} / \mathrm{L}, P<$ 0.001 ). The cutoff value of preoperative serum albumin for postoperative AKI occurrence was $37.5 \mathrm{~g} / \mathrm{L}$ determined by the Youden index $(\mathrm{P}<0.001$, area under the

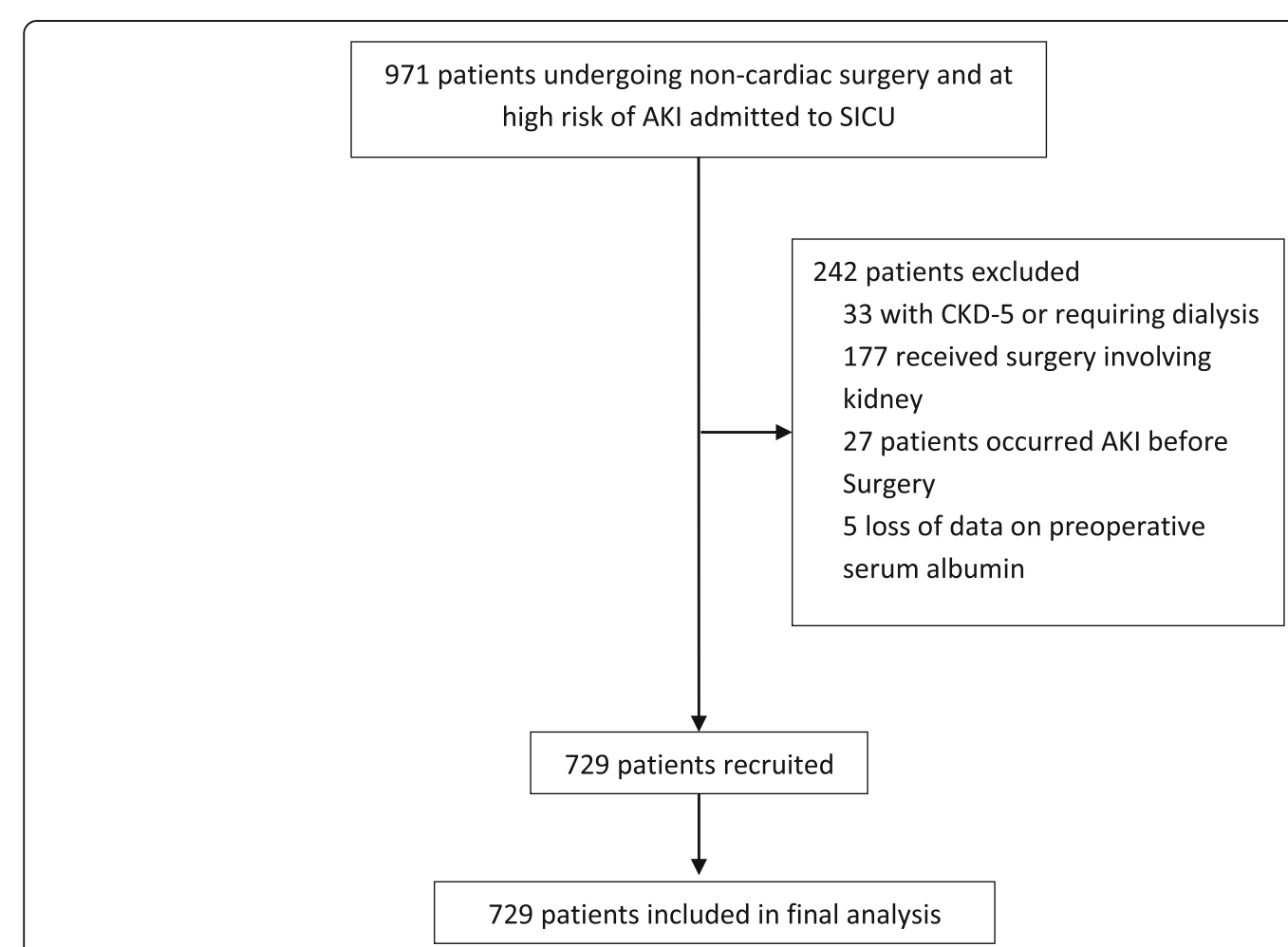

Fig. 1 Flow diagram of the study 
Table 1 Preoperative variables

\begin{tabular}{|c|c|c|c|c|c|c|c|}
\hline & $\begin{array}{l}\text { Total } \\
(n=729)\end{array}$ & $\begin{array}{l}\text { Without postoperative AKI } \\
(n=541)\end{array}$ & $\begin{array}{l}\text { With postoperative AKI } \\
(n=188)\end{array}$ & $P$ value & $\begin{array}{l}\text { Preoperative albumin } \geq 37.5 \mathrm{~g} / \mathrm{L}^{\mathrm{a}} \\
(n=456)\end{array}$ & $\begin{array}{l}\text { Preoperative albumin }<37.5 \mathrm{~g} / \mathrm{L}^{\mathrm{a}} \\
(n=273)\end{array}$ & $P$ value \\
\hline Age $(y)$ & $67 \pm 16$ & $66 \pm 16$ & $71 \pm 14$ & $<0.001$ & $66 \pm 15$ & $69 \pm 14$ & 0.056 \\
\hline Male sex & $432(59.3 \%)$ & $318(58.8 \%)$ & $114(60.6 \%)$ & 0.655 & $276(60.5 \%)$ & $156(57.1 \%)$ & 0.368 \\
\hline $\mathrm{BMI}\left(\mathrm{kg} / \mathrm{m}^{2}\right)$ & $24.1 \pm 4.5$ & $24.0 \pm 4.6$ & $24.6 \pm 4.4$ & 0.139 & $24.7 \pm 4.3$ & $23.2 \pm 4.7$ & $\begin{array}{l}< \\
0.001\end{array}$ \\
\hline \multicolumn{8}{|l|}{ Preoperative comorbidities } \\
\hline Diabetes mellitus & $194(26.6 \%)$ & $136(25.1 \%)$ & $58(30.9 \%)$ & 0.127 & $123(27.0 \%)$ & $71(26.0 \%)$ & 0.775 \\
\hline Hypertension & $399(54.7 \%)$ & $287(53.0 \%)$ & $112(59.6 \%)$ & 0.122 & $260(57.0 \%)$ & $139(50.9 \%)$ & 0.109 \\
\hline Coronary heart disease & $195(26.7 \%)$ & $138(25.5 \%)$ & $57(30.3 \%)$ & 0.199 & $130(28.5 \%)$ & $65(23.8 \%)$ & 0.165 \\
\hline Congestive heart failure & $23(3.2 \%)$ & $14(2.6 \%)$ & $9(4.8 \%)$ & 0.133 & $13(2.9 \%)$ & $10(3.7 \%)$ & 0.544 \\
\hline Cerebrovascular disease & $140(19.2 \%)$ & $99(18.3 \%)$ & $41(21.8 \%)$ & 0.293 & $94(20.6 \%)$ & $46(16.8 \%)$ & 0.212 \\
\hline Chronic kidney disease & $23(3.2 \%)$ & $9(1.7 \%)$ & $14(7.4 \%)$ & $<0.001$ & $8(1.8 \%)$ & $15(5.5 \%)$ & 0.005 \\
\hline Lung disease ${ }^{b}$ & $68(9.3 \%)$ & $51(9.4 \%)$ & $17(9.0 \%)$ & 0.876 & $41(9.0 \%)$ & $27(9.9 \%)$ & 0.686 \\
\hline Liver disease $^{c}$ & $20(2.7 \%)$ & $16(3.0 \%)$ & $4(2.1 \%)$ & 0.548 & $10(2.2 \%)$ & $10(3.7 \%)$ & 0.240 \\
\hline Malignant neoplasm & $474(65.0 \%)$ & $349(64.5 \%)$ & $125(66.5 \%)$ & 0.624 & $247(65.1 \%)$ & $177(64.8 \%)$ & 0.935 \\
\hline Peripheral vascular disease & $37(5.1 \%)$ & $24(4.4 \%)$ & $13(6.9 \%)$ & 0.182 & $21(4.6 \%)$ & $16(5.9 \%)$ & 0.455 \\
\hline ASA classification & & & & $<0.001$ & & & $\begin{array}{l}< \\
0.001\end{array}$ \\
\hline I & $12(1.6 \%)$ & $12(2.2 \%)$ & $0(0.0 \%)$ & & $7(1.5 \%)$ & $5(1.8 \%)$ & \\
\hline$\|$ & $275(37.7 \%)$ & $224(41.4 \%)$ & $51(27.1 \%)$ & & $202(44.3 \%)$ & $73(26.7 \%)$ & \\
\hline III & $404(55.4 \%)$ & $284(52.5 \%)$ & $120(63.8 \%)$ & & $238(52.2 \%)$ & $166(60.8 \%)$ & \\
\hline IV & $38(5.2 \%)$ & $21(3.9 \%)$ & $17(9.0 \%)$ & & $9(2.0 \%)$ & $29(10.6 \%)$ & \\
\hline Preoperative $\mathrm{Hb}(g / L)^{d}$ & $124 \pm 23$ & $125 \pm 22$ & $119 \pm 26$ & 0.007 & $133 \pm 18$ & $108 \pm 23$ & $\begin{array}{l}< \\
0.001\end{array}$ \\
\hline Preoperative albumin $(g / L)^{d}$ & $38.6 \pm 6.2$ & $39.3 \pm 6.0$ & $36.7 \pm 6.3$ & $<0.001$ & $42.5 \pm 3.0$ & $32.1 \pm 4.2$ & $\begin{array}{l}< \\
0.001\end{array}$ \\
\hline $\begin{array}{l}\text { Preoperative albumin }<37.5 \\
\mathrm{~g} / \mathrm{L}\end{array}$ & $273(37.4 \%)$ & $175(32.3 \%)$ & $98(52.1 \%)$ & $<0.001$ & - & - & - \\
\hline $\begin{array}{l}\text { Baseline serum creatinine } \\
(\text { umol/L) })^{\mathrm{e}}\end{array}$ & $80.4 \pm 26.3$ & $78.2 \pm 22.2$ & $86.7 \pm 35.1$ & 0.002 & $81.5 \pm 23.6$ & $78.5 \pm 30.4$ & 0.163 \\
\hline Preoperative BNP $(\mathrm{pg} / \mathrm{ml})^{\mathrm{f}}$ & $98(43,190)$ & $84(38,166)$ & $126(61,220)$ & 0.001 & $66(31,133)$ & $140(72,257)$ & $\begin{array}{l}< \\
0.001\end{array}$ \\
\hline Radiocontrast exposure $^{9}$ & $76(10.4 \%)$ & $48(8.9 \%)$ & $28(14.9 \%)$ & 0.020 & $44(9.6 \%)$ & $32(11.7 \%)$ & 0.375 \\
\hline On ACEl/ARB & $62(8.5 \%)$ & $48(8.9 \%)$ & $14(7.4 \%)$ & 0.546 & $38(8.3 \%)$ & $24(8.8 \%)$ & 0.830 \\
\hline Smoking habit ${ }^{\mathrm{h}}$ & $136(18.7 \%)$ & $108(20.0 \%)$ & $28(14.9 \%)$ & 0.124 & $94(20.6 \%)$ & $42(15.4 \%)$ & 0.079 \\
\hline
\end{tabular}

Data are presented as mean $\pm \mathrm{SD}$, median (interquartile range), or number of patients (percentage) and compared by independent samples t-test, Mann-Whitney $\mathrm{U}$ test or chi-squared test/ Fisher's exact test respectively

$A C E I$ angiotensin converting enzyme inhibitor, ARB angiotensin receptor blocker, ASA American Society of Anesthesiologists, BMI body mass index, BNP B-type natriuretic peptide, cTNI cardiotroponin, $\mathrm{Hb}$ hemoglobin

${ }^{a}$ The cutoff value of preoperative albumin for postoperative AKI was determined by the Youden index of the ROC curve [see Additional file 3: Figure S1]

Including chronic obstructive pulmonary disease, asthma, and pulmonary fibrosis

Including any kind of chronic hepatitis and liver cirrhosis

${ }^{\mathrm{d}}$ Measured within 3 days before surgery

eDetermined by the minimal value of serum creatinine measured within 3 months before admission and in hospital before surgery; if neither value was available, the modification of diet in renal disease formula was adopted to estimate the baseline serum creatinine according to the Kidney Disease Improving Global Outcomes guideline

${ }^{\mathrm{f}}$ Measured in 366 patients before surgery

Including patients who had radiocontrast exposure within 7 days before surgery

${ }^{h}$ Smoking for more than 10 cigarettes per day for more than 1 year, including current or past smokers

curve $[\mathrm{AUC}]=0.624$ ) with a sensitivity of 0.54 , specificity of 0.67 , and positive predictive value of 0.36 [see Additional file 3: Figure S1].

The incidence of AKI in patients with serum albumin $<37.5 \mathrm{~g} / \mathrm{L}(35.9 \%$ [98/273]) was significantly higher than those with serum albumin $\geq 37.5 \mathrm{~g} / \mathrm{L}(19.7 \%$ [90/456]) $(P<0.001)$. Univariate analysis showed that preoperative serum albumin $<37.5 \mathrm{~g} / \mathrm{L}$ was strongly associated with the occurrence of postoperative AKI (OR 2.277; 95\% CI 1.624-3.194; $\mathrm{P}<0.001)$. In the multivariate logistic regression model (backward), preoperative serum albumin
$<37.5 \mathrm{~g} / \mathrm{L}$ was identified to be independently associated with postoperative AKI (OR 1.892; 95\% CI 1.238-2.891; $\mathrm{P}<0.001)$. Other independent risk factors for AKI included age (OR 1.018; 95\% CI 1.004-1.033; $P=0.013$ ), radiocontrast exposure (OR 1.843; 95\% CI 1.031-3.293; $P=0.039$ ), baseline creatinine (OR 1.016; 95\% CI 1.0081.025; $P<0.001$ ), ASA classification (OR 1.719; 95\% CI $1.193-2.477 ; P=0.004)$, and intraoperative use of vasopressors (OR 1.680; 95\% CI 1.065-2.648; $P=0.026$ ) (Table 3). After matching for age, BMI, history of chronic kidney disease, preoperative hemoglobin, and 
Table 2 Intra- and postoperative variables

\begin{tabular}{|c|c|c|c|c|c|c|c|}
\hline & $\begin{array}{l}\text { Total } \\
(n=729)\end{array}$ & $\begin{array}{l}\text { Without } \\
\text { postoperative AKI } \\
(n=541)\end{array}$ & $\begin{array}{l}\text { With postoperative } \\
\text { AKI }(n=188)\end{array}$ & $\begin{array}{l}P \\
\text { value }\end{array}$ & $\begin{array}{l}\text { Preoperative albumin } \\
\geq 37.5 \mathrm{~g} / \mathrm{L}^{\mathrm{a}}(n=456)\end{array}$ & $\begin{array}{l}\text { Preoperative albumin }< \\
37.5 \mathrm{~g} / \mathrm{L}^{\mathrm{a}}(n=273)\end{array}$ & $\begin{array}{l}P \\
\text { value }\end{array}$ \\
\hline $\begin{array}{l}\text { Duration of anesthesia } \\
\text { (min) }\end{array}$ & $\begin{array}{l}258(174, \\
338)\end{array}$ & $253(169,337)$ & $277(192,347)$ & 0.053 & $243(167,330)$ & $272(201,364)$ & 0.002 \\
\hline $\begin{array}{l}\text { Duration of surgery } \\
\text { (min) }\end{array}$ & $\begin{array}{l}171(97 \\
249)\end{array}$ & $165(92,245)$ & $192(110,256)$ & 0.062 & $158(88,242)$ & $189(121,272)$ & 0.002 \\
\hline Emergency surgery & $64(8.8 \%)$ & $37(6.8 \%)$ & $27(14.4 \%)$ & 0.002 & $17(3.7 \%)$ & $47(17.2 \%)$ & $\begin{array}{l}< \\
0.001\end{array}$ \\
\hline Open surgery ${ }^{b}$ & $288(53.9 \%)$ & $193(50.0 \%)$ & 95 (64.2\%) & 0.003 & $147(45.1 \%)$ & $141(67.8 \%)$ & $\begin{array}{l}< \\
0.001\end{array}$ \\
\hline \multicolumn{8}{|l|}{ Type of surgery } \\
\hline General surgery $^{c}$ & $330(45.3 \%)$ & $229(42.3 \%)$ & $101(53.7 \%)$ & 0.007 & $180(39.5 \%)$ & $150(54.9 \%)$ & $\begin{array}{l}< \\
0.001\end{array}$ \\
\hline Neurosurgery & $18(2.5 \%)$ & $15(2.8 \%)$ & $3(1.6 \%)$ & 0.585 & $10(2.2 \%)$ & $8(2.9 \%)$ & 0.535 \\
\hline Thoracic surgery & $52(7.1 \%)$ & $38(7.0 \%)$ & $14(7.4 \%)$ & 0.846 & $33(7.2 \%)$ & $19(7.0 \%)$ & 0.888 \\
\hline Urologic surgery & $195(26.7 \%)$ & $162(29.9 \%)$ & $33(17.6 \%)$ & 0.001 & $160(35.1 \%)$ & 35 (12.8\%) & $\begin{array}{l}< \\
0.001\end{array}$ \\
\hline Gynecological surgery & $25(3.4 \%)$ & 17 (3.1\%) & $8(4.3 \%)$ & 0.470 & $6(1.3 \%)$ & 19 (7.0\%) & $\begin{array}{l}< \\
0.001\end{array}$ \\
\hline Orthopedic surgery & $52(7.1 \%)$ & $38(7.0 \%)$ & $14(7.4 \%)$ & 0.846 & $29(6.4 \%)$ & $23(8.4 \%)$ & 0.294 \\
\hline Vascular surgery & $15(2.1 \%)$ & $10(1.8 \%)$ & $5(2.7 \%)$ & 0.552 & $11(2.4 \%)$ & $4(1.5 \%)$ & 0.383 \\
\hline ENT surgery & $8(1.1 \%)$ & $6(1.1 \%)$ & $2(1.1 \%)$ & $\begin{array}{l}> \\
0.999\end{array}$ & $3(0.7 \%)$ & $5(1.8 \%)$ & 0.158 \\
\hline Others ${ }^{d}$ & $24(3.3 \%)$ & $18(3.3 \%)$ & $6(3.2 \%)$ & 0.928 & $18(3.9 \%)$ & $6(2.2 \%)$ & 0.200 \\
\hline $\begin{array}{l}\text { Intraoperative maximal } \\
\text { lactate }(\mathrm{mmol} / \mathrm{L})^{\mathrm{e}}\end{array}$ & $1.1(0.8,1.5)$ & $1.1(0.8,1.5)$ & $1.1(0.8,1.6)$ & 0.995 & $1.1(0.8,1.5)$ & $1.1(0.8,1.5)$ & 0.421 \\
\hline $\begin{array}{l}\text { Intraoperative minimal } \\
\mathrm{Hb}(\mathrm{g} / \mathrm{L})^{\mathrm{e}}\end{array}$ & $112 \pm 26$ & $113 \pm 26$ & $111 \pm 27$ & 0.568 & $118 \pm 25$ & $104 \pm 25$ & $\begin{array}{l}< \\
0.001\end{array}$ \\
\hline \multicolumn{8}{|l|}{ Intraoperative management } \\
\hline Use of vasopressors ${ }^{f}$ & $175(24.1 \%)$ & $116(21.6 \%)$ & $59(31.4 \%)$ & 0.007 & $116(21.6 \%)$ & $59(31.4 \%)$ & 0.007 \\
\hline $\begin{array}{l}\text { Volume of artificial } \\
\text { colloid infusion (ml) }\end{array}$ & $\begin{array}{l}500(500 \\
1000)\end{array}$ & $500(500,1000)$ & $500(500,1000)$ & 0.858 & $500(500,1000)$ & $500(500,1000)$ & 0.618 \\
\hline $\begin{array}{l}\text { Estimated blood loss } \\
(\mathrm{ml})\end{array}$ & $\begin{array}{l}100(10 \\
300)\end{array}$ & $100(10,300)$ & $100(10,300)$ & 0.138 & $100(10,300)$ & $100(10,400)$ & 0.034 \\
\hline $\begin{array}{l}\text { Positive fluid balance } \\
(\mathrm{ml})\end{array}$ & $2202 \pm 1605$ & $2201 \pm 1579$ & $2204 \pm 1679$ & 0.982 & $2093 \pm 1516$ & $2382 \pm 1731$ & 0.023 \\
\hline \multicolumn{8}{|c|}{ Postoperative variables before AKI ${ }^{g}$} \\
\hline \multicolumn{8}{|l|}{ Nephrotoxin exposure } \\
\hline Glycopeptides & $46(6.3 \%)$ & $35(6.5 \%)$ & $11(5.9 \%)$ & 0.764 & $17(3.7 \%)$ & $29(10.6 \%)$ & $\begin{array}{l}< \\
0.001\end{array}$ \\
\hline Aminoglycoside & $0(0.0 \%)$ & $0(0.0 \%)$ & $0(0.0 \%)$ & - & $0(0.0 \%)$ & $0(0.0 \%)$ & - \\
\hline NSAIDs & $387(53.1 \%)$ & $292(54.0 \%)$ & $95(50.5 \%)$ & 0.415 & $265(58.1 \%)$ & $122(44.7 \%)$ & $\begin{array}{l}< \\
0.001\end{array}$ \\
\hline Hemolysis & $1(0.1 \%)$ & $1(0.2 \%)$ & $0(0.0 \%)$ & $\begin{array}{l}> \\
0.999\end{array}$ & $0(0.0 \%)$ & $1(0.4 \%)$ & 0.374 \\
\hline Rhabdomyolysis & $1(0.1 \%)$ & $1(0.2 \%)$ & $0(0.0 \%)$ & $\begin{array}{l}> \\
0.999\end{array}$ & $0(0.0 \%)$ & $1(0.4 \%)$ & 0.374 \\
\hline Sepsis & $47(6.4 \%)$ & $29(5.4 \%)$ & $18(9.6 \%)$ & 0.043 & $12(2.6 \%)$ & 35 (12.8\%) & $\begin{array}{l}< \\
0.001\end{array}$ \\
\hline Use of vasopressors ${ }^{f}$ & 77 (10.6\%) & $53(9.8 \%)$ & $24(12.8 \%)$ & 0.254 & $41(9.0 \%)$ & $36(13.2 \%)$ & 0.074 \\
\hline Minimal $\mathrm{Hb}(\mathrm{g} / \mathrm{L})$ & $103 \pm 25$ & $105 \pm 26$ & $99 \pm 21$ & 0.008 & $109 \pm 27$ & $94 \pm 18$ & $\begin{array}{l}< \\
0.001\end{array}$ \\
\hline
\end{tabular}


Table 2 Intra- and postoperative variables (Continued)

\begin{tabular}{|c|c|c|c|c|c|c|c|}
\hline & $\begin{array}{l}\text { Total } \\
(n=729)\end{array}$ & $\begin{array}{l}\text { Without } \\
\text { postoperative AKI } \\
(n=541)\end{array}$ & $\begin{array}{l}\text { With postoperative } \\
\text { AKI }(n=188)\end{array}$ & $\begin{array}{l}P \\
\text { value }\end{array}$ & $\begin{array}{l}\text { Preoperative albumin } \\
\geq 37.5 \mathrm{~g} / \mathrm{L}^{\mathrm{a}}(n=456)\end{array}$ & $\begin{array}{l}\text { Preoperative albumin }< \\
37.5 \mathrm{~g} / \mathrm{L}^{\mathrm{a}}(n=273)\end{array}$ & $\begin{array}{l}P \\
\text { value }\end{array}$ \\
\hline Maximal BNP (pg/ml) & $\begin{array}{l}247(130, \\
456)\end{array}$ & $244(128,443)$ & $264(142,513)$ & 0.265 & $209(115,373)$ & $322(188,564)$ & $\begin{array}{l}< \\
0.001\end{array}$ \\
\hline $\begin{array}{l}\text { Maximal lactate (mmol/ } \\
\text { L) }\end{array}$ & $1.9(1.3,2.7)$ & $1.9(1.3,2.7)$ & $1.8(1.3,2.8)$ & 0.751 & $1.9(1.4,2.7)$ & $1.8(1.3,2.7)$ & 0.377 \\
\hline $\begin{array}{l}\text { Perioperative blood } \\
\text { transfusion }^{h}\end{array}$ & $186(25.5 \%)$ & $131(24.2 \%)$ & 55 (29.3\%) & 0.172 & 89 (19.5\%) & 97 (35.5\%) & $\begin{array}{l}< \\
0.001\end{array}$ \\
\hline $\begin{array}{l}\text { Non-renal SOFA within } \\
24 \mathrm{~h} \mathrm{ICU} \text { admission }\end{array}$ & $2(1,3)$ & $2(1,3)$ & $2(2,4)$ & 0.005 & $2(1,3)$ & $3(1,4)$ & 0.001 \\
\hline Postoperative AKI & $188(25.8 \%)$ & - & - & - & 90 (19.7\%) & 98 (35.9\%) & $\begin{array}{l}< \\
0.001\end{array}$ \\
\hline
\end{tabular}

Data are presented as mean $\pm \mathrm{SD}$, median (interquartile range), or number of patients (percentage) and compared by independent samples t-test, Mann-Whitney $\mathrm{U}$ test or chi-squared test/Fisher's exact test respectively

$B N P$ B-type natriuretic peptide, ENT ear, nose and throat, $\mathrm{Hb}$ hemoglobin, NSAIDs non-steroidal anti-inflammatory drugs, SOFA sequential organ failure assessment score

${ }^{\text {a }}$ The cutoff value of preoperative albumin for postoperative AKI was determined by the Youden index of the ROC curve [see Additional file 3: Figure S1]

${ }^{b}$ Open or laparoscopic surgery referred to 534 patients

c Abdominal surgery, such as gastrointestinal, hepatobiliary, and pancreatic surgery

d Thyroid or breast surgery

${ }^{\mathrm{e}}$ Measured by arterial blood gas analysis

${ }^{f}$ Including use of phenylephrine, norepinephrine, epinephrine, and dopamine

${ }^{g}$ Occurred before start of AKI

${ }^{h}$ Perioperative blood product transfusion, including packed red blood cell, plasma, and platelet

ASA classification, 161 pairs of patients with or without hypoalbuminemia were well balanced in their baseline variables except for malignant neoplasm and ASA classification [see Additional file 4: Table S3; Additional file 5: Table S4]. Logistic regression analysis once again revealed that preoperative serum albumin $<37.5 \mathrm{~g} / \mathrm{L}$ was independently associated with postoperative AKI (OR 3.085; 95\% CI 1.649-5.771; $P<0.001$ ) [see Additional file 6: Table S5].

Moreover, for severity of AKI, patients with preoperative serum albumin $<37.5 \mathrm{~g} / \mathrm{L}$ tended to have a higher but not significant ratio in AKI stage $2(2.6 \%$ vs $1.1 \%$, $P=0.144)$ and a much higher ratio in AKI stage $3(4.8 \%$ vs $0.7 \%, P<0.001)$ than those with preoperative serum albumin $\geq 37.5 \mathrm{~g} / \mathrm{L}$.

To determine the cause of hypoalbuminemia, we further analyzed preoperative nutritional status using criteria of nutritional risk screening 2002 (NRS 2002). The results showed that patients with preoperative serum albumin $<37.5 \mathrm{~g} / \mathrm{L}$ had significantly increased NRS score $[4(2,4)$ vs $1(1,2), P<0.001]$ and had a much higher ratio of NRS score $\geq 3(77.5 \%$ vs $15.5 \%, P<0.001)$.

Of all included patients, 14 patients $(1.9 \%)$ died during hospital stay. Patients with preoperative serum albumin < $37.5 \mathrm{~g} / \mathrm{L}$ had a mortality rate of $4.4 \%$, which was much higher than $0.4 \%$ in patients with preoperative serum albu$\min \geq 37.5 \mathrm{~g} / \mathrm{L}(P<0.001)$. The cumulative survival rate was also lower in patients with hypoalbuminemia $(P=0.003)$ (Fig. 2). Compared with that in non-AKI patients, the mortality rate was significantly higher in AKI patients $(6.9 \%$ [13/188] vs. $0.2 \%$ [1/541]; $\mathrm{P}<0.001)$. Kaplan-Meier analysis revealed that the cumulative survival rate decreased with increasing AKI severity $(P<0.001)$ (Fig. 3$)$. In addition, postoperative AKI was associated with other worse outcomes, such as prolonged mechanical ventilation $[53.4(33.0,73.8)$ vs $14.7(11.1,18.3)$ hours, $P<0.001]$, higher rate of other postoperative complications $[0(0,2)$ vs $0(0,0), P<0.001]$, ICU stay $[4.0(3.1,4.9)$ vs $2.0(1.8,2.3)$ days, $P<0.001]$, postoperative hospital stay $[17.8(14.8,20.9)$ vs $12.3(11.3$, 13.3) days, $P<0.001]$, and higher total cost $[13,453$ (8538, $20,228)$ vs $11,306(6277,16,400)$ dollars, $P<0.001$ ] (Table 4 , Additional file 2: Table S2). We also further analyzed AKI patients, preoperative hypoalbuminemia $(<37.5 \mathrm{~g} / \mathrm{L})$ was associated with more use of MV (72.4\% [71/98] vs. $56.7 \%$ [51/90]; $P=0.024)$, longer ICU stay $[4.5(3.3,5.7)$ vs 3.4 $(2.1,4.8)$ days, $P=0.027$, higher occurrence of postoperative complications $[1(0,3)$ vs $0(0,1), \mathrm{P}<0.001]$, and higher mortality $(11.2 \%[11 / 98]$ vs. $2.2 \%[2 / 90] ; P=0.020)$ and total cost $[15,160(10,345,22,221)$ vs $12,111(6262,17,763)$ dollars, $P=0.011$ ] (Table 4). All the dataset of our study are available [see Additional file 7: Dataset].

\section{Discussion}

Results of this retrospective study showed that preoperative hypoalbuminemia was independently associated with AKI occurrence in high-risk patients following non-cardiac surgery. In addition, more severe AKI stage was found in hypoalbuminemic patients. In accordance with previous reports of outcomes after non-cardiac surgery $[1,2,20]$, the in-hospital mortality rate in AKI patients $(6.9 \%)$ was very much higher than that in patients without AKI (0.2\%). Other outcomes, such as ICU, 
Table 3 Independent risk factors for postoperative AKI

\begin{tabular}{|c|c|c|c|c|}
\hline & $\underline{\text { Univariate Logistic m }}$ & & Multivariate Logistic & \\
\hline & $\mathrm{OR}(95 \% \mathrm{Cl})$ & $P$ value & OR $(95 \% \mathrm{Cl})$ & $P$ value \\
\hline Age (y) & $1.022(1.010-1.034)$ & $<0.001$ & $1.018(1.004-1.033)$ & 0.013 \\
\hline History of chronic kidney disease & $4.756(2.023-11.180)$ & $<0.001$ & - & - \\
\hline Sepsis & $1.869(1.013-3.451)$ & 0.046 & - & - \\
\hline Radiocontrast exposure $^{c}$ & $1.797(1.091-2.961)$ & 0.021 & $1.843(1.031-3.293)$ & 0.039 \\
\hline Baseline creatinine $(u m o l / L){ }^{d}$ & $1.011(1.005-1.017)$ & $<0.001$ & $1.016(1.008-1.025)$ & $<0.001$ \\
\hline Preoperative $\mathrm{Hb}(\mathrm{g} / \mathrm{L})^{e}$ & $0.990(0.983-0.997)$ & 0.004 & - & - \\
\hline Preoperative albumin $<37.5 \mathrm{~g} / \mathrm{L}^{\mathrm{e}}$ & $2.277(1.624-3.194)$ & $<0.001$ & $1.892(1.238-2.891)$ & 0.003 \\
\hline ASA classification & $1.966(1.472-2.625)$ & $<0.001$ & $1.719(1.193-2.477)$ & 0.004 \\
\hline Open surgery & $1.792(1.213-2.650)$ & 0.003 & - & - \\
\hline Emergency surgery & $2.284(1.349-3.869)$ & 0.002 & & \\
\hline General surgery ${ }^{f}$ & $1.582(1.133-2.208)$ & 0.007 & - & - \\
\hline Duration of surgery (every $1 \mathrm{~h}$ increase) & $1.047(0.976-1.124)$ & 0.202 & - & - \\
\hline Intraoperative use of vasopressors ${ }^{9}$ & $1.660(1.146-2.404)$ & 0.007 & $1.680(1.065-2.648)$ & 0.026 \\
\hline Non-renal SOFA within $24 \mathrm{~h} \mathrm{ICU}$ admission & $1.133(1.044-1.230)$ & 0.003 & - & - \\
\hline 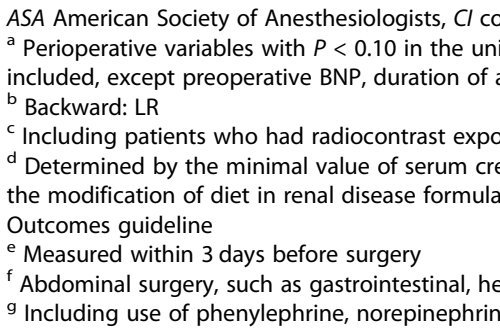 & $\begin{array}{l}\text { terval, } H b \text { hemoglobin, } \\
\text { lyses by independent sar } \\
\text { and postoperative minim } \\
7 \text { days before surgery } \\
\text { asured within } 3 \text { months } \\
\text { ed to estimate the baseli } \\
\text { and pancreatic surgery } \\
\text { ine, and dopamine }\end{array}$ & $\begin{array}{l}\text { SOFA sec } \\
\text { Mann-Wh } \\
\text { AKI beca } \\
\text { sion and } \\
\text { atinine a }\end{array}$ & $\begin{array}{l}\text { jan failure assessment } \mathrm{sc} \\
\text {, chi-squared test, or Fis } \\
\text { earity } \\
\text { oefore surgery; if neither } \\
\text { the Kidney Disease Imp }\end{array}$ & $\begin{array}{l}\text { est were } \\
\text { available, } \\
\text { al }\end{array}$ \\
\hline
\end{tabular}

postoperative hospital stay, and total cost, were also much worse in AKI patients. Furthermore, AKI patients with hypoalbuminemia had even more detrimental outcomes.

Cumulative evidence have shown that hypoalbuminemia is an important risk factor for postoperative AKI in various clinical settings [8-12]. However, in surgical settings, studies were mainly focused on cardiac surgery and transplant surgery $[11,12,21-23]$. Few studies have examined the effect of preoperative hypoalbuminemia on postoperative AKI patients undergoing non-cardiac surgery. Kim et al. [15] conducted a retrospective study enrolling 4718 patients who underwent partial or total gastrectomy for gastric cancer, and they revealed that patients with preoperative hypoalbuminemia, defined as $<40 \mathrm{~g} / \mathrm{L}$, had a significantly increased risk for AKI (OR 1.4; 95\% CI 1.11-1.77). In patients following hip fracture surgery or total knee arthroplasty, after adjustment for confounders, early postoperative hypoalbuminemia has been shown to be strongly associated with AKI with a cutoff value of $<29 \mathrm{~g} / \mathrm{L}$ and $<30 \mathrm{~g} / \mathrm{L}[16,17]$. Recently, Kim et al. found that a preoperative serum albumin level $<38 \mathrm{~g} / \mathrm{L}$ was independently associated with AKI (OR 2.465; CI 1.310-4.640) and mortality (OR 3.223; CI 1.959-5.305) in patients undergoing brain tumor surgery
[18]. The finding from our study that preoperative hypoalbuminemia had a significant relationship with postoperative AKI was consistent with the results above. The cutoff value for hypoalbuminemia in our patients was $37.5 \mathrm{~g} / \mathrm{L}$, well above the usually accepted definition for hypoalbuminemia. Thus, our results suggested that with even a little decrease in preoperative serum albumin concentration, a higher incidence of postoperative AKI would occur in high-risk patients undergoing non-cardiac surgery.

Several possible mechanisms underlie this association. As a scavenger of radical oxygen species, combined with its anti-inflammation effects, albumin limits tubular cell apoptosis [24, 25]. Recent data have suggested that the integrity of the glycocalyx might be compromised in patients with hypoalbuminemia leading to loss of oncotic pressure gradients and barrier function, fluid leakage into the interstitium, and microvascular flow alterations [26, 27]. Moreover, ligation of endogenous toxin, modulation of nitric oxide and pharmacokinetic and pharmacodynamic effects of albumin also play an important role in renal protection [28, 29].

Albumin cutoff values vary between studies, and we attributed this difference to various study populations 


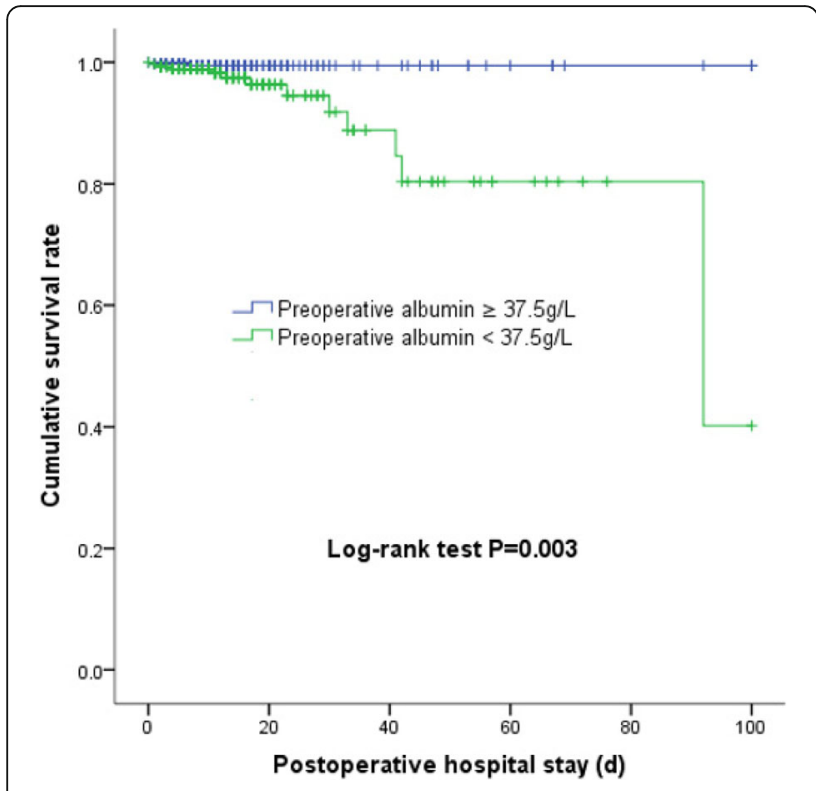

Fig. 2 Cumulative survival rate of patients with preoperative serum albumin $<37.5$ or $\geq 37.5 \mathrm{~g} / \mathrm{L}$.

and types of surgery. In our study, as mentioned above, the cutoff value of $37.5 \mathrm{~g} / \mathrm{L}$ had a sensitivity of 0.54 , specificity of 0.67 , and positive predictive value of 0.36 ; in patients undergoing brain tumor surgery, the cutoff value of $38 \mathrm{~g} / \mathrm{L}$ had a similar sensitivity of 0.54 , but lower specificity of 0.27 and positive predictive value of 0.04 , which might be partly explained by the low incidence of AKI (1.8\%) [18]. However, the AUC appeared to be similar with 0.624 in our non-cardiac surgery patients, 0.653

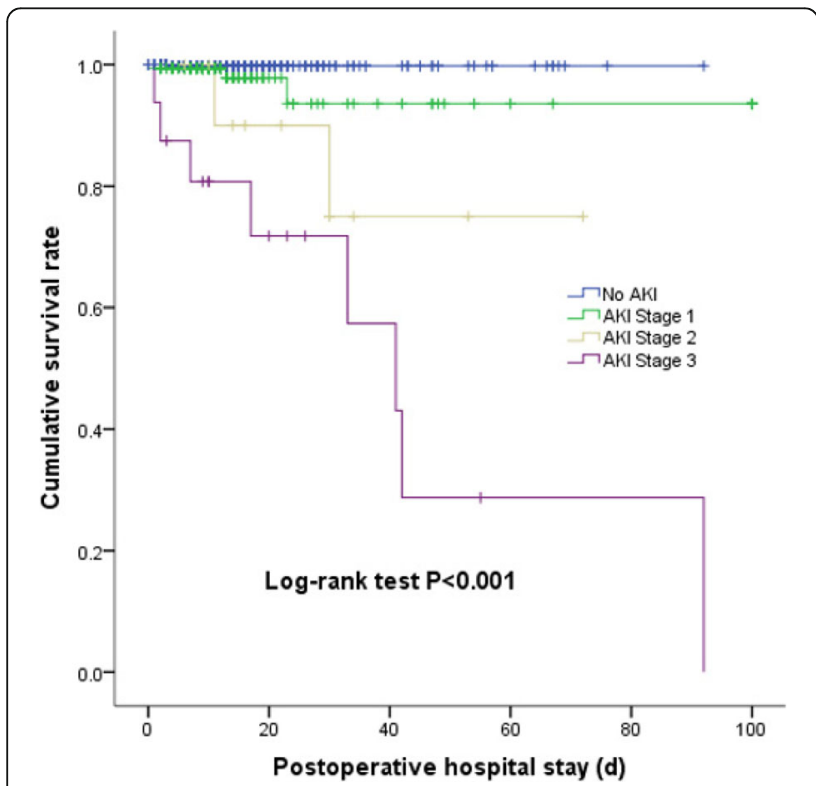

Fig. 3 Cumulative survival rate of patients with stages $1-, 2$, and 3 AKI during postoperative hospital stay in hip fracture patients [16], and 0.684 in brain tumor patients [18]. Considering the possible negative association between serum albumin and AKI occurrence as reflected by research in patients undergoing cardiac surgery [11], we assumed that patients with a higher risk of postoperative AKI, such as having several comorbidities or undergoing general surgery [4], might have a lower tolerance threshold of serum albumin for AKI occurrence, thus requiring higher levels of serum albumin to protect perioperative renal function.

Currently, increasing amount of data revealed that postoperative AKI occurrence is associated with shortterm adverse outcomes such as higher mortality and longer ICU and hospital stay $[1,2,4,11]$, which was also confirmed by our study. Furthermore, in AKI patients, preoperative hypoalbuminemia was associated with more use of MV, longer ICU stay, higher occurrence of postoperative complications, and higher mortality and total cost.

Unfortunately, there is still no effective treatment for AKI at present. Therefore, early recognition of high-risk patients and prevention of postoperative AKI become the first priority in clinical practice. Basic and clinical studies mentioned above indicated a potential benefit of correcting hypoalbuminemia for renal protection. Excitingly, Lee et al. [30] had made a step further. They recently performed a randomized controlled trial evaluating the effects of exogenous $20 \%$ human albumin solution vs saline on the incidence of postoperative AKI in adult patients with hypoalbuminemia $(<40 \mathrm{~g} / \mathrm{L})$ undergoing off-pump coronary artery bypass surgery. Their results have demonstrated that the incidence of postoperative AKI was lower in the intervention group than in the control group $(17.6 \%$ vs $31.7 \% ; P=0.031)$. Multivariate logistic regression analysis revealed a renalprotective effect of albumin infusion with nearly $60 \%$ risk of AKI decreased $(\mathrm{OR}=0.42,95 \% \mathrm{CI}$ : $0.21-0.83$; $P=0.012)$. However, further studies are needed to address the results in the future, especially in patients undergoing non-cardiac surgery. Another way to increase preoperative serum albumin level is optimization of nutritional status. As shown in our study, $77.5 \%$ of hypoalbuminemic patients had preoperative NRS score $\geq$ 3 , which indicated that malnutrition might be an important contributor to the occurrence of hypoalbuminemia. Until now, several studies have demonstrated significantly better results in overall and infectious complications in patients undergoing preoperative nutritional therapy [31-33]. However, data on the association between nutritional support and postoperative AKI were limited. Therefore, more work is needed to verify the effects of optimizing nutritional status on AKI, especially for patients undergoing non-cardiac surgery. 
Table 4 Postoperative outcomes

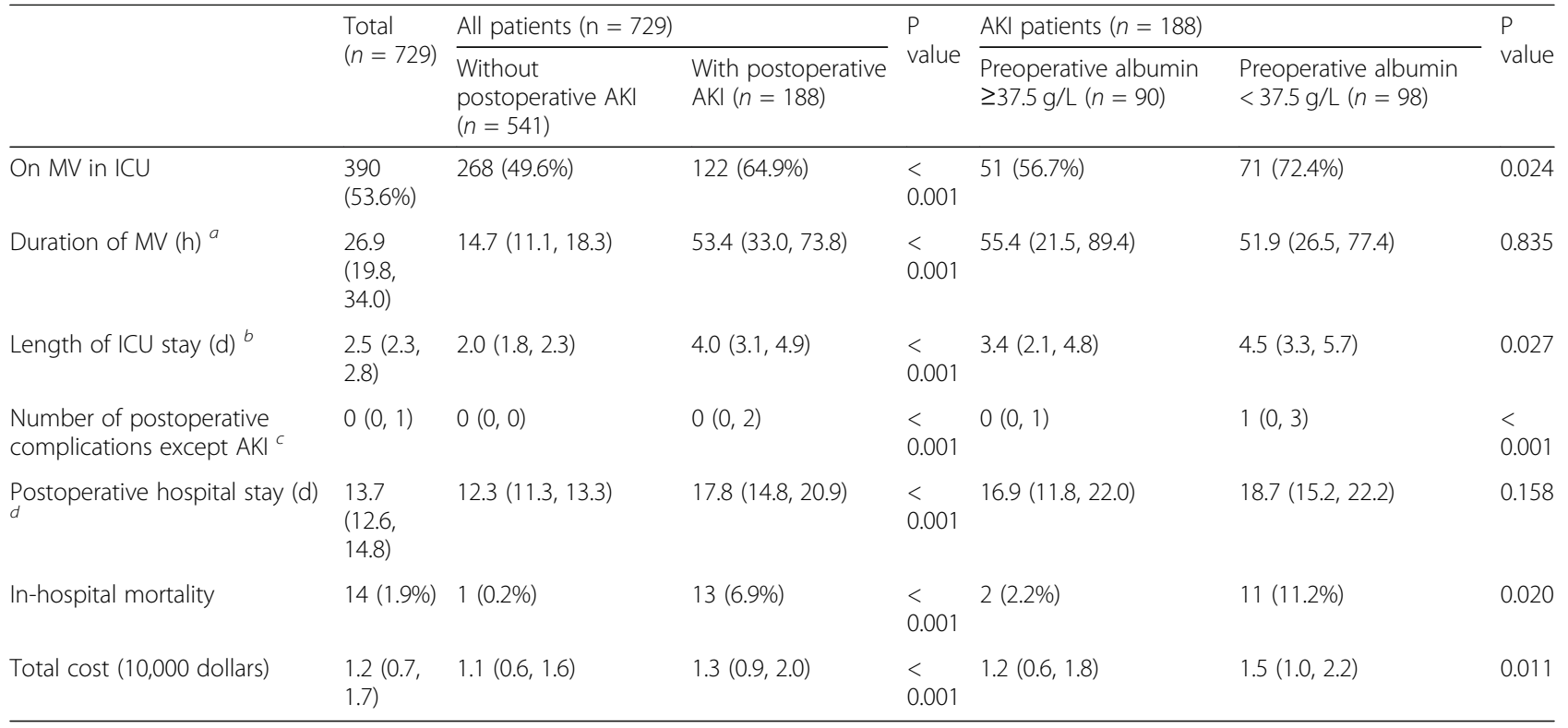

Data are presented as number of patients (percentage), or median (interquartile range), unless otherwise indicated

ICU intensive care unit, $M V$ mechanical ventilation

${ }^{a}$ Results of patients requiring postoperative mechanical ventilation. Data were analyzed by Kaplan-Meier analysis and compared by log-rank test; results are presented as average ( $95 \%$ confidence interval);

${ }^{b}$ Result of patients admitted to ICU. Data were analyzed by Kaplan-Meier analysis and compared by log-rank test; results are presented as average (95\% confidence interval)

' Including pulmonary infection, pleural effusion, atelectasis, respiratory failure, acute myocardial infarction, congestive heart failure, new-onset arrhythmia, hemodynamic insufficiency, stroke, venous thromboembolism, ileus, intra-abdominal abscess, wound infection, urinary tract infection, sepsis, surgical bleeding, digestive tract bleeding, acute liver injury, disseminated intravascular coagulation, anastomotic leakage, and wound dehiscence [see Additional file 2: Table S2] ${ }^{d}$ Data were analyzed by Kaplan-Meier analysis and compared by log-rank test; results are presented as average (95\% confidence interval)

This study has major limitations. First, although we considered many perioperative AKI-related variables in our analysis, the effects of non-investigated factors could not be totally excluded. Second, given the lack of statistical power, subgroup analyses for the association of different preoperative albumin levels with AKI were not performed. Finally, in view of the retrospective and observational nature of this study, a causal relationship between preoperative hypoalbuminemia and risk of postoperative AKI could not be determined.

\section{Conclusions}

Our results showed that preoperative hypoalbuminemia was independently associated with AKI in high-risk patients following non-cardiac surgery, and postoperative AKI was associated with adverse prognosis. Prospective trials are needed to further identify the association between hypoalbuminemia and AKI and explore the potential beneficial effects of albumin infusion or specific nutritional therapy on postoperative AKI prevention.

\section{Additional files}

Additional file 1: Table S1. STROBE Statement. Checklist of our cohort study, which demonstrates STROBE Statement Checklist of our cohort study. (DOCX $28 \mathrm{~kb})$
Additional file 2: Table S2. Occurrence of other postoperative complications. Demonstrates the occurrence and definitions of other postoperative complications. (DOCX $21 \mathrm{~kb}$ )

Additional file 3: Figure S1. Preoperative serum albumin receiver operating characteristic curve for discriminating critically ill subjects with or without AKI. Demonstrates preoperative serum albumin receiver operating characteristic curve for discriminating patients with or without AKl. (DOCX $29 \mathrm{~kb}$ )

Additional file 4: Table S3. Preoperative variables after propensity score matching. Description: Demonstrates preoperative variables between patients with or without hypoalbuminemia after propensity score matching. (DOCX $20 \mathrm{~kb}$ )

Additional file 5: Table S4. Intra- and postoperative variables after propensity score-matching. Demonstrates intra- and postoperative variables between patients with or without hypoalbuminemia after propensity score-matching. (DOCX $21 \mathrm{~kb}$ )

Additional file 6: Table S5. Independent risk factors for postoperative AKl after propensity score matching. Demonstrates the independent risk factors for postoperative AKI after propensity score matching. (DOCX $16 \mathrm{~kb}$ )

Additional file 7: Dataset. Relevant data underlying the main results. (XLSX $413 \mathrm{~kb})$

\section{Abbreviations}

AKI: Acute kidney injury; ASA: American Society of Anesthesiology; AUC: Area under the curve; BMI: Body mass index; BNP: B-type natriuretic peptide; MV: Mechanical ventilation; NRS: Nutritional risk screening; SICU: Surgical intensive care unit; SOFA: Sequential organ failure assessment 


\section{Acknowledgements}

The authors gratefully acknowledge Dr. Ning Han, Dr. Run-Xia Cheng, and Dr. Lin-Lin Yang (Department of Nephrology, Peking University First Hospital, Beijing, China) for their suggestions of the CRF design.

\section{Authors' contributions}

$\mathrm{NL}$ : study development, data collection, first draft of the manuscript, and data editing; HQ: data collection, manuscript editing; JFG: data collection and manuscript editing; HYY: data collection; XYL: statistical analysis; SLL: study design and final version of the manuscript; DXW: study design and manuscript editing; LY: study design and final version of the manuscript. All authors read and approved the final manuscript.

\section{Funding}

No funding support this research.

\section{Availability of data and materials}

All data generated or analyzed during this study are included in this published article and its supplementary information files.

\section{Ethics approval and consent to participate}

The study protocol was approved by the Clinical Research Ethics Committee of Peking University First Hospital (2018-137). The ethics committee agreed to waive the written informed consent.

\section{Consent for publication}

Not applicable.

\section{Competing interests}

The authors declare that they have no competing interests.

\section{Author details}

${ }^{1}$ Department of Critical Care Medicine, Peking University First Hospital, No.8 Xishiku Street, Xicheng District, Beijing 100034, China. ${ }^{2}$ Critical Care Nephrology Research Center, Peking University First Hospital, No.8 Xishiku Street, Xicheng District, Beijing 100034, China. ${ }^{3}$ Department of Anesthesiology, Fuwai Hospital, Chinese Academy of Medical Sciences, No.167 North Lishi Road, Xicheng District, Beijing 100037, China. ${ }^{4}$ Clinical Laboratory, Peking University First Hospital, No.8 Xishiku Street, Xicheng District, Beijing 100034, China. ${ }^{5}$ Department of Biostatistics, Peking University First Hospital, No.8 Xishiku Street, Xicheng District, Beijing 100034, China. ${ }^{6}$ Department of Nephrology, Peking University First Hospital, No.8 Xishiku Street, Xicheng District, Beijing 100034, China.

\section{Received: 2 April 2019 Accepted: 26 August 2019} Published online: 02 September 2019

\section{References}

1. Long TE, Helgason D, Helgadottir S, Palsson R, Gudbjartsson T, Sigurdsson $\mathrm{GH}$, et al. Acute kidney injury after abdominal surgery: incidence, risk factors, and outcome. Anesth Analg. 2016;122:1912-20.

2. O'Connor ME, Kirwan CJ, Pearse RM, Prowle JR. Incidence and associations of acute kidney injury after major abdominal surgery. Intensive Care Med. 2016;42:521-30.

3. Ichai C, Vinsonneau C, Souweine B, Armando F, Canet E, Clec'h C, et al. Acute kidney injury in the perioperative period and in intensive care units (excluding renal replacement therapies). Ann Intensive Care. 2016;6:48.

4. Grams ME, Sang Y, Coresh J, Ballew S, Matsushita K, Molnar MZ, et al. Acute kidney injury after major surgery: a retrospective analysis of veterans health administration data. Am J Kid Dis. 2016;67:872-80.

5. Gameiro J, Neves JB, Rodrigues N, Bekerman C, Melo MJ, Pereira M, et al. Acute kidney injury, long term renal function and mortality in patients undergoing major abdominal surgery: a cohort analysis. Clin Kidney J. 2016; 9:192-200.

6. Joannidis M, Druml W, Forni LG, Groeneveld ABJ, Honore PM, Hoste E, et al. Prevention of acute kidney injury and protection of renal function in the intensive care unit: update 2017 : Expert opinion of the Working Group on Prevention, AKI section, European Society of Intensive Care Medicine. Intensive Care Med. 2017;43:730-49.
7. Vincent JL, Dubois MJ, Navickis RJ, Wilkes MM. Hypoalbuminemia in acute illness: is there a rationale for intervention? A meta-analysis of cohort studies and controlled trials. Ann Surg. 2003;237:319-34.

8. Wiedermann CJ, Wiedermann W, Joannidis M. Causal relationship between hypoalbuminemia and acute kidney injury. World J Nephrol. 2017;6:176-87.

9. Prakash J, Gupta T, Prakash S, Rathore SS, Usha S. Acute kidney injury in patients with human immunodeficiency virus infection. Indian J Nephrol. 2015;25:86-90.

10. Hsu CY, Huang YH, Su CW, Lin HC, Chiang JH, Lee PC, et al. Renal failure in patients with hepatocellular carcinoma and ascites undergoing transarterial chemoembolization. Liver Int. 2010;30:77-84.

11. Lee EH, Baek SH, Chin JH, Choi DK, Son HJ, Kim WJ, et al. Preoperative hypoalbuminemia is a major risk factor for acute kidney injury following offpump coronary artery bypass surgery. Intensive Care Med. 2012:38:1478-86.

12. Sang BH, Bang JY, Song JG, Hwang GS. Hypoalbuminemia within two postoperative days is an independent risk factor for acute kidney injury following living donor liver transplantation: a propensity score analysis of 998 consecutive patients. Crit Care Med. 2015;43:2552-61.

13. Wratten ML, Sereni L, Tetta C. Oxidation of albumin is enhanced in the presence of uremic toxins. Ren Fail. 2001;23:563-71.

14. Wiedermann CJ, Joannidis M. Nephroprotective potential of human albumin infusion: a narrative review. Gastroenterol Res Pract. 2015;2015:912839.

15. Kim CS, Oak CY, Kim HY, Kang YU, Choi JS, Bae EH, et al. Incidence, predictive factors, and clinical outcomes of acute kidney injury after gastric surgery for gastric cancer. PLoS One. 2013;8:e82289.

16. Shin $\mathrm{KH}, \mathrm{Han} \mathrm{SB}$. Early postoperative hypoalbuminemia is a risk factor for postoperative acute kidney injury following hip fracture surgery. Injury. 2018;49:1572-6.

17. Kim HJ, Koh WU, Kim SG, Park HS, Song JG, Ro YJ, et al. Early postoperative albumin level following total knee arthroplasty is associated with acute kidney injury: a retrospective analysis of 1309 consecutive patients based on kidney disease improving global outcomes criteria. Medicine (Baltimore). 2016;95:e4489.

18. Kim K, Bang JY, Kim SO, Kim S, Kim JU, Song JG. Association of preoperative hypoalbuminemia with postoperative acute kidney injury in patients undergoing brain tumor surgery: a retrospective study. J Neurosurg. 2018; 128:1115-22.

19. Kellum JA, Lameire N, Aspelin P, Barsoum RS, Mehta RL, Burdmann EA, et al. KDIGO clinical practice guideline for acute kidney injury 2012. Kidney Int. 2012;Suppl 2:1-138.

20. Gameiro J, Fonseca JA, Neves M, Jorge S, Lopes JA. Acute kidney injury in major abdominal surgery: incidence, risk factors, pathogenesis and outcomes. Ann Intensive Care. 2018 9;8:22.

21. Kim WH, Park MH, Kim HJ, Lim HY, Shim HS, Sohn JT, et al. Potentially modifiable risk factors for acute kidney injury after surgery on the thoracic aorta: a propensity score matched casecontrol study. Medicine (Baltimore). 2015;94:e273.

22. Findik $\mathrm{O}$, Aydin U, Baris $\mathrm{O}$, Parlar $\mathrm{H}$, Alagoz GA, Ata $Y$, et al. Preoperative low serum albumin levels increase the requirement of renal replacement therapy after cardiac surgery. Heart Surg Forum. 2016;19:E123-7.

23. Moore J, He X, Shabir S, Hanvesakul R, Benavente D, Cockwell P, et al. Development and evaluation of a composite risk score to predict kidney transplant failure. Am J Kidney Dis. 2011;57:744-51.

24. Quinlan GJ, Mumby S, Martin GS, Bernard GR, Gutteridge JM, Evans TW. Albumin influences total plasma antioxidant capacity favorably in patients with acute lung injury. Crit Care Med. 2004;32:755-9.

25. Bauer M, Huse K, Settmacher U, Claus RA. The heme oxygenase-carbon monoxide system: regulation and role in stress response and organ failure. Intensive Care Med. 2008;34:640-8.

26. Schiefer J, Lebherz-Eichinger D, Erdoes G, Berlakovich G, Bacher A, Krenn $C G$, et al. Alterations of endothelial glycocalyx during orthotopic liver transplantation in patients with end-stage liver disease. Transplantation. 2015;99:2118-23.

27. Woodcock TE, Woodcock TM. Revised Starling equation and the glycocalyx model of transvascular fluid exchange: an improved paradigm for prescribing intravenous fluid therapy. Br J Anaesth. 2012;108:384-94.

28. Ha CE, Bhagavan NV. Novel insights into the pleiotropic effects of human serum albumin in health and disease. Biochim Biophys Acta. 1830;2013:5486-93.

29. Ghuman J, Zunszain PA, Petitpas I, Bhattacharya AA, Otagiri M, Curry S. Structural basis of the drug-binding specificity of human serum albumin. J Mol Biol. 2005;353:38-52. 
30. Lee EH, Kim WJ, Kim JY, Chin JH, Choi DK, Sim JY, et al. Effect of exogenous albumin on the incidence of postoperative acute kidney injury in patients undergoing off-pump coronary artery bypass surgery with a preoperative albumin level of less than $4.0 \mathrm{~g} / \mathrm{dl}$. Anesthesiology. 2016:124:1001-11.

31. Dreznik Y, Horesh N, Gutman M, Gravetz A, Amiel I, Jacobi H, et al. Preoperative nutritional optimization for Crohn's disease patients can improve surgical outcome. Dig Surg. 2018;35:442-7.

32. Wang H, Zuo L, Zhao J, Dong J, Li Y, Gu L, et al. Impact of preoperative exclusive enteral nutrition on postoperative complications and recurrence after bowel resection in patients with active Crohn's disease. World I Surg. 2016:40:1993-2000.

33. Fukuda Y, Yamamoto K, Hirao M, Nishikawa K, Maeda S, Haraguchi N, et al. Prevalence of malnutrition among gastric cancer patients undergoing gastrectomy and optimal preoperative nutritional support for preventing surgical site infections. Ann Surg Oncol. 2015;22(Suppl 3):S778-85.

\section{Publisher's Note}

Springer Nature remains neutral with regard to jurisdictional claims in published maps and institutional affiliations.

Ready to submit your research? Choose BMC and benefit from:

- fast, convenient online submission

- thorough peer review by experienced researchers in your field

- rapid publication on acceptance

- support for research data, including large and complex data types

- gold Open Access which fosters wider collaboration and increased citations

- maximum visibility for your research: over $100 \mathrm{M}$ website views per year

At BMC, research is always in progress.

Learn more biomedcentral.com/submissions 